\title{
Röntgenthorax Indikationen, Limitationen, Befundung
}

Tobias Achenbach

Der Röntgenthorax ist die am häufigsten durchgeführte Röntgenuntersuchung. Ärzte nahezu jeder Disziplin müssen sie im Rahmen ihrer Tätigkeit korrekt einsetzen und interpretieren. Besonders wichtig ist es, akute, potenziell lebensbedrohliche Veränderungen zu erkennen, um relevante therapeutische Entscheidungen daraus abzuleiten. Man muss allerdings auch die Limitationen dieser projektionsradiografischen Methode kennen - so kann man die Diagnostik rechtzeitig ergänzen.

Tab. 1
Röntgenthorax: häufige Indikationen
- thorakaler Schmerz
- Dyspnoe
- Husten
- Entzündungs- konstellation
- B-Symptomatik (Fie- ber, Nachtschweiß, Gewichtsverlust)
- Verschlechterung des Allgemeinzustands
- Dysphagie

\section{Voraussetzungen}

Fachkunde Strahlenschutz In Deutschland regelt die Röntgenverordnung den Betrieb von medizinischen Röntgengeräten. Von besonderer Bedeutung sind hierbei §23 (Indikationsstellung) und $\S 24$ (berechtigte Personen). Danach darf die konventionelle Röntgendiagnostik nur von einem fachkundigen Arzt angeordnet und befundet werden. Das bedeutet:

- Der verantwortliche, anfordernde Arzt muss über die Fachkunde Strahlenschutz verfügen (Minimalanforderung: Fachkunde Strahlenschutz „Notfalldiagnostik“)

- und eine rechtfertigende Indikation stellen.

Eigeninitiative gefragt Während die Fachkunde Strahlenschutz für Ärzte in radiologischen Kliniken und Instituten bereits früh obligat ist, bleibt sie in allen anderen Disziplinen oft der Eigeninitiative des Assistenzarztes überlassen. Ohne die notwendige Fachkunde darf aber - gemäß der Röntgenverordnung - kein Röntgenbild indiziert, angefertigt und befundet werden!

Welche Voraussetzungen für die Erlangung der Fachkunde nötig sind, steht in der Röntgenverordnung und in der „Richtlinie über die im Strahlenschutz erforderliche Fachkunde“. Auch die Ärztekammern helfen hier weiter.

\section{Indikationen \\ $\nabla$}

Bei entsprechender Symptomatik früh indiziert Am Beginn der ärztlichen Untersuchung stehen weiterhin die Erhebung der Anamnese, die Auskultation und Perkussion des Thorax.

- Zur Erstellung der rechtfertigenden Indikation für einen Röntgenthorax gehört die Abwägung von Risiko und zu erwartendem Nutzen für den Patienten.

Im Vergleich zu vielen anderen Röntgenuntersuchungen ist die effektive Dosis für einen Röntgenthorax relativ gering $(0,01-1 \mathrm{mSv})$, weil man eine Belichtungsautomatik verwendet. Deshalb ist der Röntgenthorax bei thorakalen und pulmonalen Symptomatiken früh angezeigt. @ Tab. 1 führt eine Auswahl der wichtigsten Indikationen auf.

Nutzen und Risiko in jedem Einzelfall abwägen Die oben genannte Nutzen-Risiko-Abwägung hängt allerdings von der individuellen Situation ab (Beschwerdeausmaß, Alter des Patienten, Vorgeschichte etc.) - und deren Einschätzung ist auch mit Subjektivität behaftet. Daher bleibt die Erstellung der rechtfertigenden Indikation eine ärztliche Gewissensentscheidung, die stets aufs Neue geprüft und gerechtfertigt werden muss.

\section{Automatische Zuweisungsmuster zu einer Rönt- genaufnahme (z. B. bei jeder Neuaufnahme, vor jeder Operation etc.) sind dementsprechend inakzeptabel.}

\section{Limitationen \\ $\nabla$}

Begrenzte Differenzierung der Strukturen Der Röntgenthorax ist ein Projektionsbild, daher wird jeder Bildpunkt durch viele anatomische Lokalisationen determiniert. Beispiel: In der Bildmitte verläuft ein Röntgenstrahl evtl. durch

- den dorsalen Weichteilmantel,

- die Wirbelsäule,

- Aorta descendens, 
- mediastinales Fettgewebe,

- Herz,

- Sternum und

- ventralen Weichteilmantel.

Hierdurch wird er abgeschwächt, bevor er auf dem Detektor einen Bildpunkt erzeugt. Eine Differenzierung dieser Strukturen ist deshalb nicht oder nur bedingt möglich ( Tab. 2). Hilfreich kann hier die seitliche Aufnahme sein.

- Als Faustregel kann gelten: Je kleiner, je weniger dicht und je zentraler ein pathologischer Prozess ist, desto eher übersieht man ihn im Röntgenthorax.

Auch viele wichtige Pathologien der Gefäße sind durch den Röntgenthorax nicht genau abzuklären. Weiterführend ist in der Regel die Computertomografie (CT) des Thorax, die z. B. bei der morphologischen Darstellung des Lungenparenchyms von keinem Verfahren übertroffen wird.

\section{Grundzüge der Befundung}

Stets nach Schema Erheben Sie Befunde immer nach einem festen Schema. Dies verhindert, dass man nur fokale Befunde beschreibt, andere relevante Befunde aber übersieht. Das Schema ist frei wählbar und soll alle Bildabschnitte beinhalten,

Z.B.:

- von innen nach außen

- von außen nach innen

- organbezogen (Herz, Lunge, Mediastinum, Rippen usw.)

Wesentlich ist die strikte Einhaltung dieses Schemas, welches man dann - ähnlich wie beim Autofahren - schon bald unbewusst einhalten und die Konzentration so auf die Befunde lenken kann.

In der Ausbildungsphase ist es hilfreich, die Befunde schriftlich festzuhalten - auch jenseits der Dokumentationspflicht. So kann man sich z. B. mit Fehlinterpretationen auseinandersetzen.

Grautöne $=$ Dichtegrade Obwohl das Röntgenbild deutlich mehr Grautöne aufweist, kann man 5 relevante Komponenten anhand ihrer Dichten unterscheiden:

1. Luft/Gas

2. Fett

3. Wasser

4. Knochen

5. Metall

Jede Struktur, die hauptsächlich aus einer dieser Komponenten aufgebaut ist und überlagerungsarm abgebildet wird, kann man der entsprechenden Dichte zuordnen und identifizieren ( 0 Tab. 3 ).

Keine vorschnelle Interpretation Wenn er im Arbeitsalltag regelmäßig Röntgenbilder des Thorax betrachtet, gelingt es auch dem Anfänger schnell, umschriebene pathologische Befunde zu

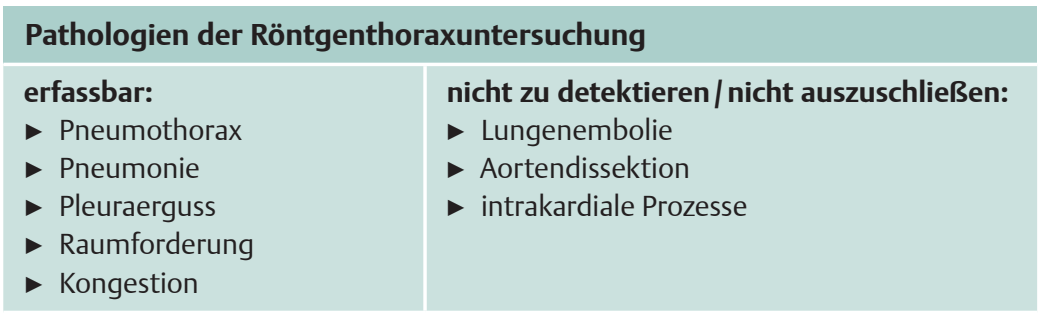

Tab. 2 Erfassbare und nicht erfassbare Pathologien in der Röntgenthoraxuntersuchung.

\begin{tabular}{|l|l|l|}
\hline \multicolumn{2}{|l|}{ Hauptdichten des konventionellen Röntgens } \\
\hline Röntgendichte & Anatomie & Pathologie \\
\hline Luft/Gas & Lunge & Pneumothorax \\
\hline Fett & $\begin{array}{l}\text { Subkutanes Fettgewebe, } \\
\text { Mamma }\end{array}$ & (großes Lipom) \\
\hline Wasser & Herz, Zwerchfell, Blut & Pleuraerguss \\
\hline Knochen & Rippen, Wirbelsäule & Verkalkungen \\
\hline Metall & & - \\
\hline
\end{tabular}

Tab. 3 Die 5 Hauptdichten des konventionellen Röntgens und ihr Vorkommen als anatomische bzw. pathologische Struktur.

\begin{tabular}{ll}
\hline Röntgentransparenz von pathologischen Befunden \\
\hline Transparenz erhöht: & Transparenz vermindert: \\
- Pneumothorax & - Pleuraerguss \\
Kaverne & - Pneumonie \\
Lungenemphysem & - Atelektase \\
Bulla & $>$ Tumor \\
& $>$ Lungenfibrose
\end{tabular}

Tab. 4 Zuordnung pathologischer Befunde zu ihrer Röntgentransparenz.

erkennen. Schwieriger ist die korrekte Interpretation. Bleiben Sie daher zunächst streng deskriptiv und gehen Sie in folgenden Schritten vor:

1. Wo ist der pathologische Befund lokalisiert?

$\triangleright$ rechts, links, beidseits

$\triangleright$ Ober-, Mittel-, Unterlappen

$\triangleright$ zentral, peripher

2. Wie ist die Transparenz des Befundes?

$\triangleright$ erhöht, erniedrigt

3. Wie stellt sich die Morphologie des Befundes dar?

$\triangleright$ homogen, inhomogen, wolkig

$\triangleright$ Volumenvermehrung, Volumenabnahme - Tab. 4 ordnet die wichtigsten Befunde ihrer Röntgentransparenz zu. Die typische Morphologie wird jeweils in den einzelnen Abschnitten behandelt.

\section{Die wichtigsten Befunde}

\section{Herzvergrößerung}

$\nabla$

Größe und Randstrukturen beachten Zur Abschätzung einer Vergrößerung der Herzsilhouette wird der kardiothorakale Quotient verwendet. 


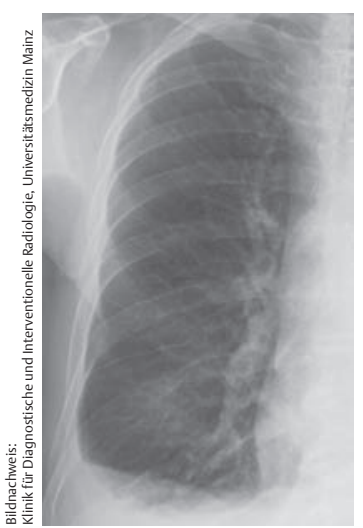

Abb. 1 Rechts normale Darstellung des lateralen Randwinkels. Auf dem linken Foto ist der (rechte) laterale Randwinkel durch einen Pleuraerguss ausgefüllt (abgerundet). Auch in den folgenden Beispielen wird sich der Pleuraerguss stets wie hier als homogene Transparenzverminderung darstellen.
Abb. 3 Liegendthorax bei beidseitigem Pleuraerguss. Der Erguss ist im Liegen dorsal angesammelt und muss erst hoch genug steigen, um eine Verschattung der Zwerchfellkonturen zu verursachen (typisches Zeichen).
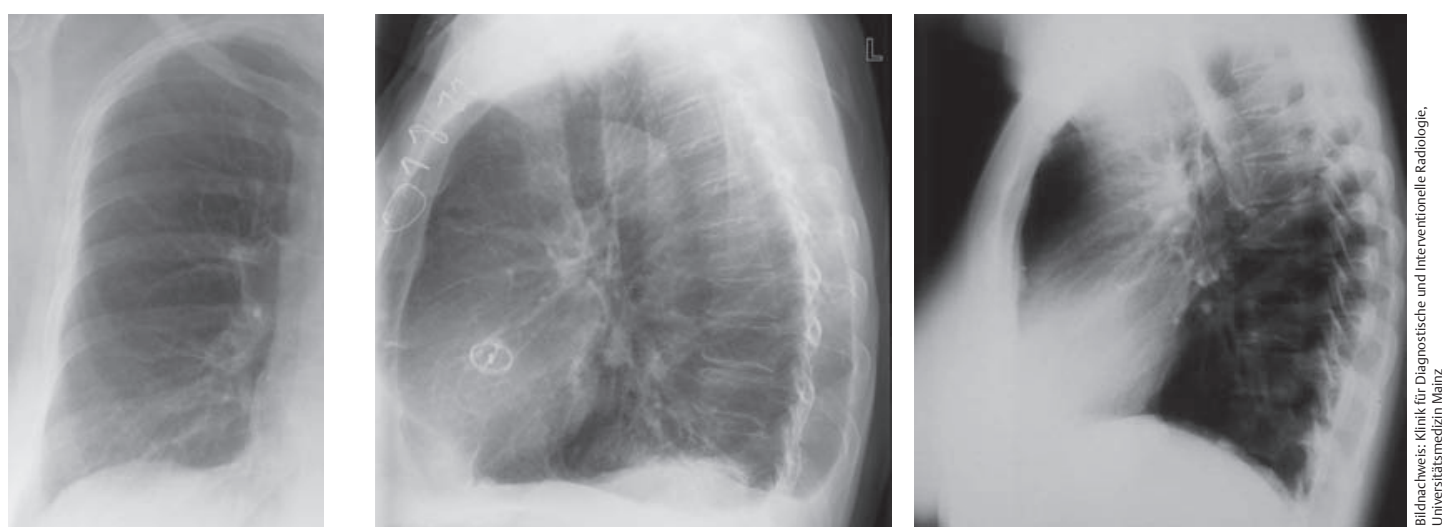

Abb. 2 Rechts normale Darstellung des dorsalen Randwinkels. Auf der linken Seite ist der laterale Randwinkel durch einen Pleuraerguss aufgefüllt und somit abgerundet.

Hierzu setzt man in der p.-a.-Aufnahme (posterior-anterior) den horizontalen maximalen Herzdurchmesser ins Verhältnis zum inneren horizontalen maximalen Lungendurchmesser.

- Werte über 0,5 gelten als pathologisch (der Herzquerdurchmesser muss also mindestens

2-mal in den Lungenquerdurchmesser passen). Die randbildenden Strukturen der Herzsilhouette werden in vielen Lehrbüchern erläutert. Ihre Kenntnis dient der Formbeurteilung. Aufgrund der Überlegenheit der Echokardiografie oder der Kardio-MRT bzw. -CT wird die Herzbeurteilung im Röntgenthorax hier nicht weiter vertieft.

\section{Pleuraerguss}

\section{$\nabla$}

Homogene Transparenzminderung Der Pleuraerguss ist eine pathologische Flüssigkeitsansammlung im Spalt zwischen Pleura visceralis und Pleura parietalis. Da sich Flüssigkeiten im Pleuraspalt frei ausbreiten können, kommt es zu einer spezifischen, sehr homogenen Transparenzminderung. Je nach Körperposition differiert die Röntgenmorphologie:

- Im Stehen werden kleine, pleurale Flüssigkeitsansammlungen zunächst im dorsalen Randwinkel an einer Abrundung sichtbar.

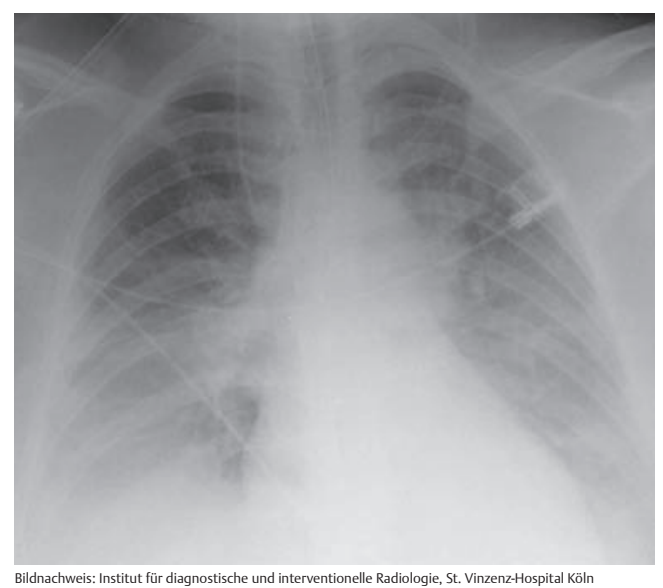

In der p.-a.-Aufnahme kommt der Pleuraerguss erst bei größerer Ausdehnung zur Darstellung zunächst im lateralen Randwinkel (Abrundung), später auch als homogene, kaudal lokalisierte Transparenzverminderung ( $\bullet$ Abb. 1 und 2 ).

- Am wenigsten sensitiv ist die Aufnahme in Rückenlage (z.B. Intensivpatienten).

Hier muss der Erguss höher steigen, um zu einer Konturauslöschung des Zwerchfells und später der Herzkontur zu führen ( $\bullet$ Abb. 3).

- In keiner Position ist der Pleuraerguss verlässlich quantifizierbar - eher noch gibt die Menge des noch ventilierten Lungenparenchyms einen Hinweis auf die klinische Relevanz.

Zeigt sich eine homogene Transparenzminderung mit glatter, konvexer Begrenzung zum Lungenparenchym (z.B. lateral oder dorsal), kann dies auf einen entzündlichen Erguss (Pleuraempyem) oder einen Hämatothorax hinweisen ( $\bullet$ Abb. 4).

\section{Infiltrat}

Inhomogene Transparenzverminderung Der Begriff Infiltrat steht im Weiteren für ein pneumonisches Infiltrat. Es handelt sich um eine alveoläre oder interstitielle Ansammlung einer entzündlichen, zellreichen Flüssigkeit.

- Dies geht mit einer inhomogenen (z.B. wolkigen) Transparenzverminderung einher.

Eines der wenigen pathognomonischen Zeichen in der konventionellen Röntgendiagnostik ist das positive Bronchopneumogramm. Es entsteht durch belüftete Atemwege, die durch das Infiltrat verlaufen und durch den daraus entstehenden Dichteunterschied sichtbar werden ( $\bullet$ Abb. 5). Infiltrate bevorzugen die basalen Lungenabschnitte, können aber in der ganzen Lunge vorkommen und verschiedene Morphologien annehmen (dicht, flau, streifig, wolkig, körnig etc.). Bei ausgedehnten, beidseitigen Infiltraten ist die schwierigste Differenzialdiagnose die Kongestion (s.u.).

- Die korrekte Diagnose hängt oft von ergänzenden und verlässlichen klinischen Angaben ab. 


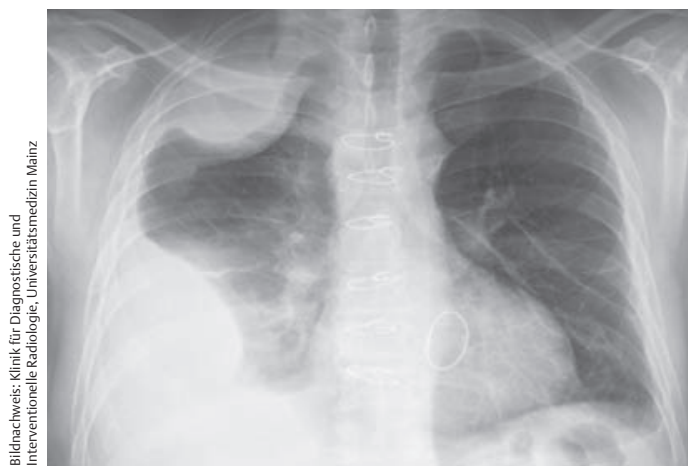

Abb. 4 Die rechtsseitigen, flüssigkeitsdichten Ergussansammlungen sind lateral und apikal gefangen und konvex zum Lungenparenchym. In diesem Fall handelte es sich um einen Hämatothorax. Eine solche Morphologie wäre aber auch typisch für ein Pleuraempyem.

\section{Pneumothorax}

$\nabla$

Lunge fällt zusammen Ein Pneumothorax entsteht, wenn Umgebungsluft in den Pleuraspalt eindringt und das zuvor zwischen Pleura visceralis und parietalis vorhandene Vakuum aufhebt.

Der Pneumothorax macht als einzige Pathologie die sonst unsichtbare Pleura visceralis der Lunge sichtbar.

In der Regel stammt diese Luft aus einer Verletzung an der Lungenoberfläche - häufig iatrogen, z. B. durch Punktion beim Legen eines ZVK oder beim Drainieren eines Pleuraergusses. Hierduch fällt die Lunge in sich zusammen, meist allerdings nicht vollständig. Gerade ein kleiner Pneumothorax entgeht dem Befunder bei flüchtiger Betrachtung (Spitzenpneumothorax).

- Befundet der Arzt anhand eines digitalen Bildes, sollte er bei dieser Fragestellung die Bildschirmlupe oder den Vergrößerungsfaktor des Programms auf den Lungenapex anwenden.

Spannungspneumothorax Ein sog. Spannungspneumothorax entsteht, wenn in der Inspiration Luft in den Pleuraspalt einströmen kann, aber bei der Exspiration nicht mehr entweicht (Ventilmechanismus, meist durch Verletzungen der Pleura visceralis). Im Röntgenbild macht er sich durch eine Volumenzunahme bemerkbar:

- Zwerchfell ipsilateral tiefstehend,

- erweiterte Interkostalräume,

- Mediastinalshift nach kontralateral (๑ Abb. 6).

Der Spannungspenumothorax ist ein Notfall, den man umgehend durch Evakuation der Luft aus dem Pleuraspalt behandeln muss.
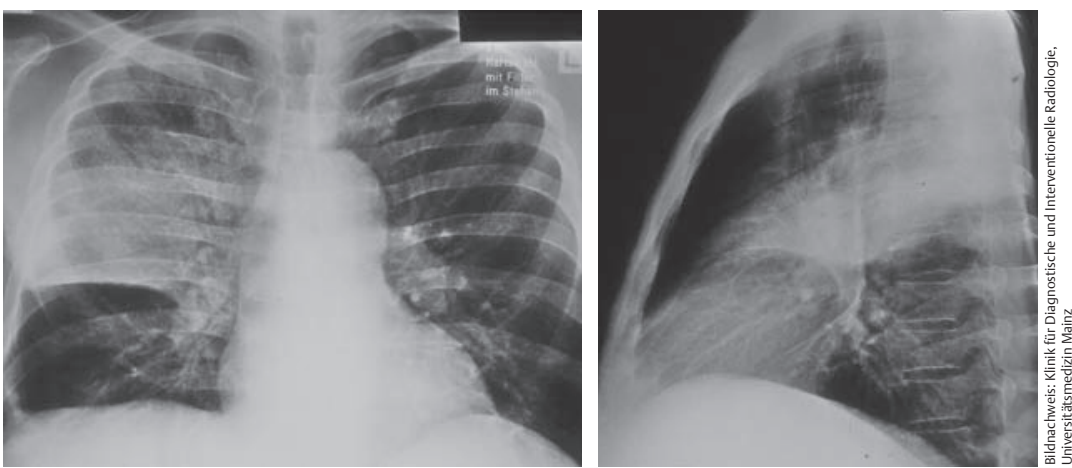

Abb. 5 Eine wolkige, inhomogene Transparenzverminderung des rechten Oberlappens. In den zentralen Lungenabschnitten werden die luftgefüllten Atemwege sichtbar, da das umgebende Lungenparenchym durch ein Infiltrat in seiner Transparenz vermindert ist. Dieser Bildeffekt das sogenannte positive Bronchopneumogramm - ist typisch für eine Pneumonie oder Infiltrate anderer Genese.

\section{Pulmonalvenöse Kongestion (pv-Stau) \\ $\nabla$}

Blutstauung Ursache der pulmonalvenösen Kongestion ist eine Linksherzinsuffizienz: Das Blut staut sich in den Lungenvenen vor dem linken Ventrikel. Zunächst führt dies im Rahmen der Volumenkapazität zu einer Vergrößerung der Lungenvenen, die im Röntgenbild dann betont zur Darstellung kommen.

- Eines der ersten Phänomene ist die basoapikale Umverteilung.

Im physiologischen Fall (stehender, gesunder Patient) sind die kaudalen Lungenvenen von stärkerem Kaliber als die apikalen. Dieses gleicht sich bei der Kongestion an. Die Veränderung kann allerdings subtil sein ( $\bullet$ Abb. 7 ).

Cave Beim Röntgenthorax im Liegen ist dieses Phänomen durch die veränderte Position schwerkraftbedingt auch ohne Kongestion zu beobachten. Bei der sogenannten Bettlunge darf man also eine basoapikale Umverteilung nicht werten.

Flüssigkeit im Interstitium Nachdem die Volumenkapazität der Lungenvenen erschöpft ist, kommt es zu einer Druckerhöhung, anschließend

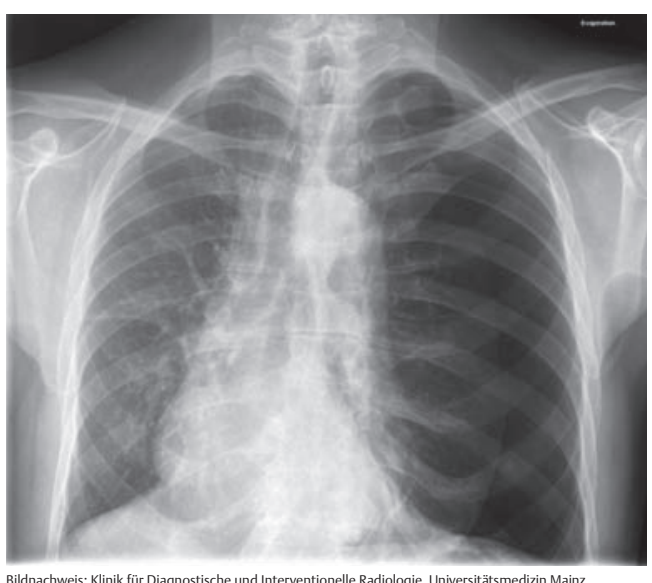

Abb. 6 Durch einen linksseitigen Pneumothorax wird die Pleura parietalis als feine, hier weitgehend parallel zur lateralen Thoraxwand verlaufende Linie sichtbar. Jenseits dieser Linie ist keine pulmonale Gefäßzeichnung mehr vorhanden. In diesem Bild zeigen sich zusätzlich alle Zeichen eines Spannungspneumothorax: Shift des Mediastinums nach kontralateral, erweiterte Interkostalräume ipsilateral, niedergedrücktes Zwerchfell ipsilateral. Notfall! 
Abb. 7 Linksherzinsuffizienter Patient bei dilatativer Kardiomyopathie (links) sowie nach diuretischer Therapie (rechts). Bei Betrachtung der Lungengefäße fällt eine Verbreiterung und Betonung der Gefäßschatten im linken Oberfeld zum Zeitpunkt der Dekompensation auf. Diese basoapikale Umverteilung ist manchmal das einzige Zeichen einer pulmonalvenösen Kongestion.

Abb. 8 Links deutliche pulmonalvenöse Kongestion und rechts Kontrolle nach erfolgreicher Therapie. Dieser pulmonalvenöse Stau zeigt sich durch eine prominente und unscharfe Darstellung der vaskulären Strukturen. Insbesondere zentral ist das gut zu erkennen.
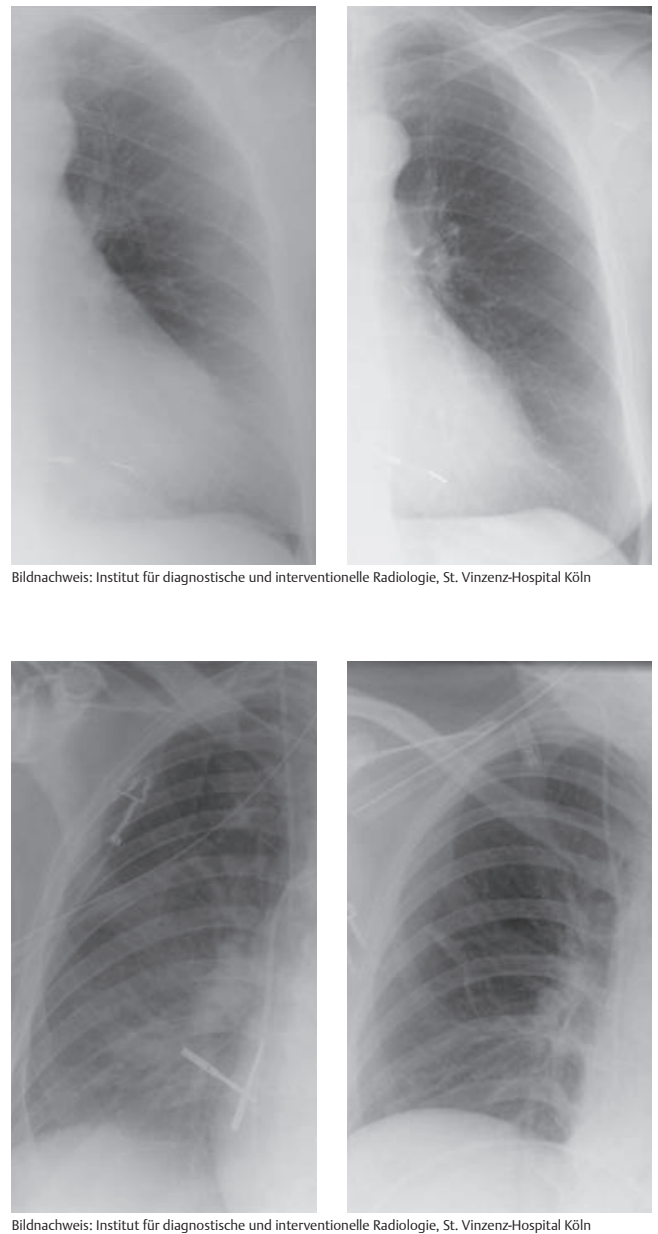

Abb. 9 Rechts Normalzustand. Links hingegen pulmonalvenöse Kongestion mit Ausbildung vieler Kerley-Linien (v. a. Kerley B, einige Kerley C). Diese können auch weniger prominent zur Darstellung kommen. Sind sie vorhanden, so ist stauungsbedingt bereits Flüssigkeit in das Interstitium übergetreten.

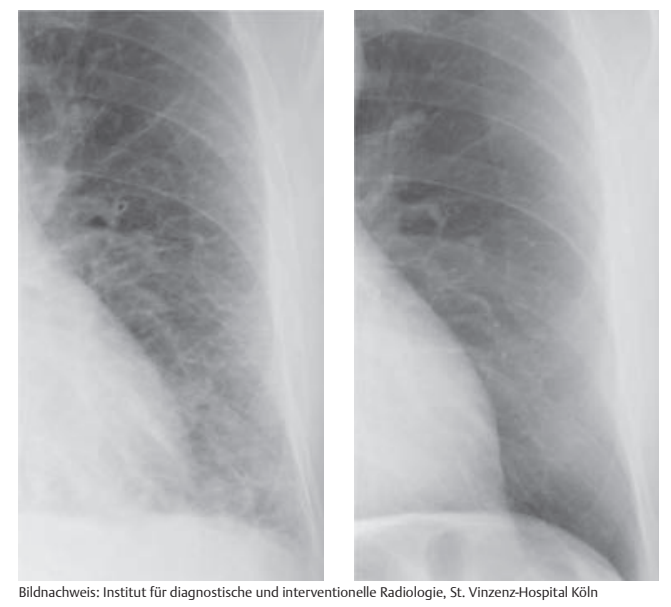

tritt die zuvor intravasale Flüssigkeit in das Interstitium über.

- Das zentrale Korrelat im Röntgenbild ist eine perihiläre Unschärfe: Der Kontrast zwischen hilusnahen Strukturen und Lungenparenchym nimmt ab, die Konturen werden unscharf (०Abb. 8).

Dieser Bildbefund kann sich beim Lungenödem zum „Schmetterlingsödem“ auswachsen.

- Das periphere Korrelat im Röntgenbild sind die sogenannten Kerley-Linien ( $\boldsymbol{\bullet}$ Abb. 9) - benannt nach dem irischen Radiologen PJ Kerley.
Die interstitielle Flüssigkeit führt zu einer Verdickung der interlobulären Septen, die im Normalfall unsichtbar sind. Liegen diese Septen parallel zum Strahlenbündel (wie z.B. der Lappenspalt im Seitbild), so werden sie als zarte, kurze, meist horizontale Linie sichtbar.

- Am häufigsten ist dieses Phänomen in den kaudalen, pleuranahen Lungenabschnitten rechts zu beobachten, hier Kerley-B-Linien genannt.

- Die gleichen Verdickungen können sich (seltener) auch apikal (Kerley-A) und (noch seltener) zentral (Kerley- $C$ ) befinden.

Übung und Erfahrung nötig Die z.T. subtilen Befunde der pulmonalvenösen Kongestion können durch verschiedene Faktoren verändert sein. So kann ein Lungenemphysem durch die zugrunde liegende Lungendestruktion die interstitiellen Zeichen erst spät und - besonders beim Lungenödem - manchmal retikulär verändert zum Vorschein kommen lassen. Dadurch wird z.B. die Morphologie einer diffusen Pneumonie imitiert. Auch Adipositas kann die Diagnose erschweren.

Um die pulmonalvenöse Kongestion sicher beurteilen zu können, braucht man viel Übung und Erfahrung. Wie bei keiner anderen akuten Veränderung ist das Bild nur in gewissenhafter Korrelation mit dem klinischen Zustand aussagekräftig.

\section{Atelektase}

\section{$\nabla$}

Oft nur in 1 Ebene sichtbar Die Atelektase bezeichnet einen kollabierten, unvollständig ausgedehnten Lungenabschnitt. Sie zeigt sich als homogene Transparenzverminderung und geht mit einer Volumenverminderung des betroffenen Areals einher (Segment, Lappen, Lungenhälfte).

- Bei kleineren Befunden ist sie häufig nur in einer Ebene zu erkennen, da sie durch den Volumenverlust meist flach ist.

In welcher Ebene die Atelektase sichtbar ist, hängt vom betroffenen Lungenabschnitt ab. In vielen „Kitteltaschenbüchern“ finden sich entsprechende Schemata, auf die hier nur verwiesen werden soll. $\bigcirc$ Abb. 10 zeigt ein Beispiel für die besonders häufige Atelektase des Mittellappens (sichtbar im Seitbild, im p.-a.-Bild unsichtbar oder nur als flaue Transparenzverminderung zu erahnen).

Auswirkung auf Nachbarstrukturen Je nach Größe wirkt sich die Atelektase auf Nachbarstrukturen aus. Eine Lappenatelektase kann das Zwerchfell oder den Hilus zu sich ziehen. Die Atelektase eines gesamten Lungenflügels kann das Mediastinum zur Atelektase hin ziehen - ein gutes Kriterium zur Unterscheidung von einem sehr großen Pleuraerguss mit Totalverschattung eines Hemithorax. 

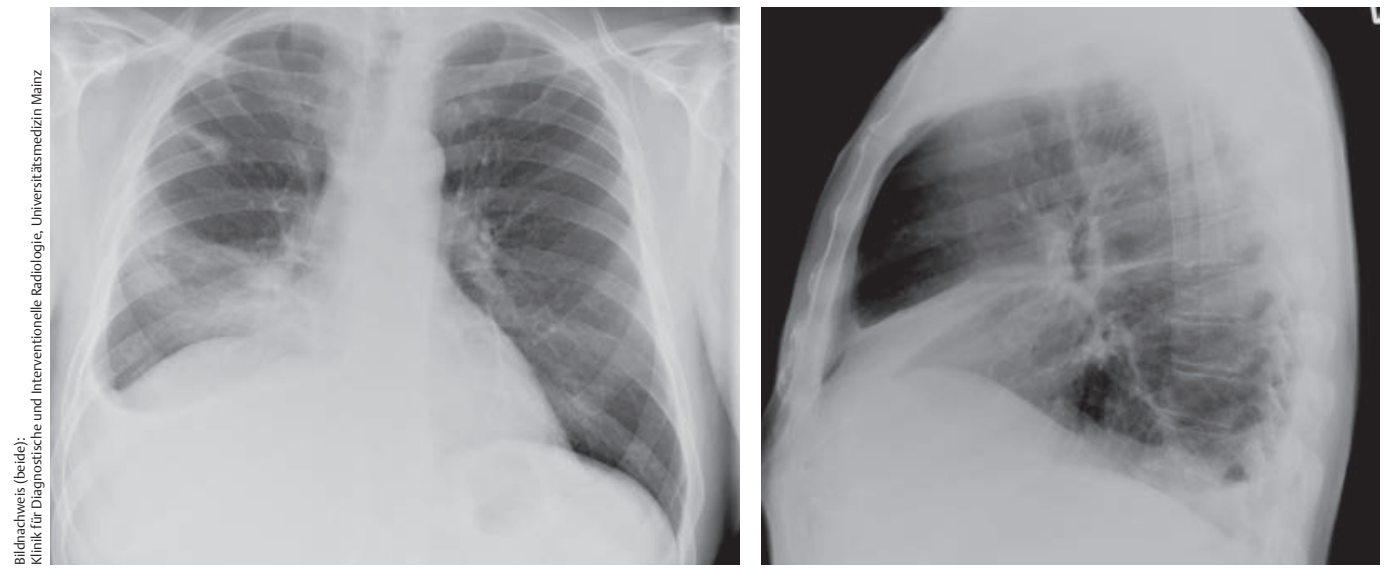

Fazit Der Röntgenthorax ist die primäre, bildgebende Methode der Wahl, wenn z. B. Pleuraerguss, Infiltrat, Pneumothorax, pulmonalvenöser Stau oder Atelektase nachgewiesen oder ausgeschlossen werden sollen. Eine systematische Analyse hilft, alle Befunde zu erfassen. Die neutrale Deskription von Lokalisation, Transparenz und Morphologie führt zur korrekten Diagnose.

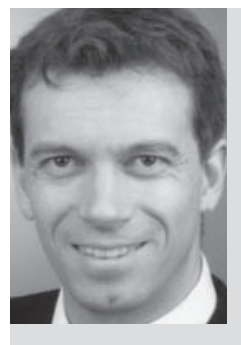

PD Dr. med. Tobias Achenbach ist Chefarzt des Instituts für diagnostische und interventionelle Radiologie am St. Vinzenz-Hospital in Köln. Er lehrt an der Radiologischen Klinik der Universitätsmedizin Mainz und ist zertifizierter Ausbilder der Dt. Gesellschaft für Interventionelle Radiologie und minimal-invasive Therapie. E-Mail: achenbac@unimainz.de

\section{Mehr zum Thema}

Verordnung über den Schutz vor Schäden durch Röntgenstrahlen (RöV)

o www.bmu.de/strahlenschutz

Hofer M. Chest-X-Ray-Trainer - Röntgen-ThoraxDiagnostik. 3. Aufl. Düsseldorf: Didamed; 2010

Felson B, Weinstein AS, Spitz HB. Röntgenologische Grundlagen der Thoraxdiagnostik - ein programmierter Text. 6. Aufl. Stuttgart: Thieme; 1984

Kirchner J. Trainer Thoraxdiagnostik - systematische kardiopulmonale Bildanalyse. Stuttgart: Thieme; 2009

Raoof S, Feigin D, Sung A et al. Interpretation of plain chest roentgenogram. Chest 2012; 141: 545-558 (Review)

Interessenkonflikt Der Autor erklärt, dass keine Interessenkonflikte vorliegen.

\section{Kernaussagen}

- Der Röntgenthorax steht nach Anamnese und körperlicher Untersuchung oft an erster Stelle der apparativen Diagnostik bei thorakalen Beschwerden.

- Wer einen Röntgenthorax indiziert und anschließend interpretiert, muss über die Fachkunde Strahlenschutz verfügen.

- Jedes Thoraxbild muss nach einem festen Schema analysiert werden, um keine relevanten Befunde zu übersehen.

- Man beschreibt zunächst die Pathologie:

$\triangleright$ Wo?

$\triangleright$ Transparenz erhöht oder vermindert?

$\triangleright$ Morphologie?

Daraus lässt sich der Befund interpretieren.

- Je kleiner, je weiter zentral/mediastinal und je im Röntgenbild des Thorax übersehen.

- Der Röntgenthorax hilft nicht weiter bei Lungenembolie, Aortendissektion und z. B. intrakardialen Befunden.

- Die Eskalation zur weiterführenden Diagnostik hängt von der individuellen Situation ab. Wie wichtig ist es im konkreten Fall, auch kleine Befunde zu erkennen? Vermute ich Erkrankungen der großen, zentralen Gefäße oder des Mediastinums?

- Die CT ist das weiterführende Verfahren, welches Aussagen zur vaskulären und mediastinalen Situation liefert und in der morphologischen Darstellung des Lungenparenchyms bisher von keinem Verfahren übertroffen wird. transparenter ein Befund ist, desto eher wird er
Abb. 10 Mittellappenatelektase. Diese zeigt im Seitbild (rechts) die charakteristische, flache, homogene, glatt berandete, keilförmige Transparenzverminderung (Pfeil) in Projektion auf die Herzsilhouette. Im p.-a.-Bild (links) hingegen ist der Befund nur als flaue Verschattung sichtbar, da die Ränder der Atelektase nicht parallel zum Strahlengang liegen. Der relative, ventral betonte Hochstand des rechten Zwerchfells ist Ausdruck der Volumenabnahme - ein wichtiges bildmorphologische Kriterium der Atelektase. Zusätzlich fällt ein Pleuraerguss des rechten lateralen Randwinkels auf. 